\title{
Latent autoimmune diabetes in adults in a population-based cohort of Polish patients with newly diagnosed diabetes mellitus
}

Barbara Szepietowska, Anna Głębocka, Urszula Puch, Maria Górska, Małgorzata Szelachowska

Department of Endocrinology, Diabetology and Internal Medicine, Medical University of Bialystok, Poland

Submitted: 27 August 2010

Accepted: 24 February 2011

Arch Med Sci 2012; 8, 3: 491-495

DOI: 10.5114/aoms.2012.29405

Copyright @ 2011 Termedia \& Banach

\section{Abstract}

Introduction: Latent autoimmune diabetes in adults (LADA) is a slowly developing form of autoimmune diabetes characterized by the presence of type 1 diabetes-associated autoantibody. The aim of this study was to determine the incidence and characteristics of LADA in a population-based cohort of Polish patients with newly-diagnosed diabetes.

Material and methods: The study cohort was taken from the resident population of the city Białystok, Poland, during the period 1 January to 31 December 2003, aged 20-64 years. During this period we identified 231 cases of diabetes. We measured glutamic acid decarboxylase (GADA) and insulin antibody (IAA), insulin, $\mathrm{C}$ peptide and glycated hemoglobin $\left(\mathrm{HbA}_{1 \mathrm{c}}\right)$. Diagnosis of LADA was made according to Immunology Diabetes Society and Action LADA criteria.

Results: The incidence of LADA was 10 per year per 100000 people. The proportion of patients with LADA was $8.9 \%$ among newly diagnosed cases with diabetes. Patients with LADA were younger at diagnosis ( $48.5 \pm 9.4$ years vs. $54.8 \pm 10.6$ years, $p<0.01)$, had lower body mass index $\left(26.9 \pm 9.3 \mathrm{~kg} / \mathrm{m}^{2}\right.$ vs. $29.5 \pm 5.2 \mathrm{~kg} / \mathrm{m}^{2}$, $p<0.05)$, C peptide (126 $\pm 127 \mathrm{pmol} / / \mathrm{vs} .446 \pm 592 \mathrm{pmol} / \mathrm{l}, p<0.001)$, and were less insulin resistant (HOMA IR $0.94 \pm 0.85$ vs. $3.6 \pm 4.4, p<0.001$ ) compared to patients with type 2 diabetes. Glycated hemoglobin and fasting glucose were similar in patients with LADA and type 2 diabetes.

Conclusions: In addition to GAD, anti-insulin antibodies are useful for diagnosing autoimmune diabetes in adults. Patients with LADA have similar glucose control parameters $\left(\mathrm{HbA}_{1 \mathrm{c}}\right)$ compared to patients with type 2 diabetes, although they are usually younger and have a lower body mass index. Patients with LADA make up a significant proportion of newly diagnosed people with diabetes mellitus in a Polish population.

Key words: latent autoimmune diabetes in adults, incidence, Poland, anti-glutamic acid decarboxylase antibody, anti-insulin autoantibodies.

\section{Introduction}

Latent autoimmune diabetes in adults (LADA) is a slowly progressing form of autoimmune diabetes, characterized by the presence of type 1 diabetes-associated autoantibody. Although a recent hypothesis proposed that LADA is a mixture of two major types of diabetes, comprising a heterogeneous population, it can be identified mainly based on the presence of auto-antibodies [1].

\author{
Corresponding author: \\ Prof. Małgorzata \\ Szelachowska MD, MD \\ Department of Endocrinology, \\ Diabetology and Internal \\ Medicine \\ Medical University \\ of Bialystok \\ 24 A M. Skłodowskiej-Curie \\ 15-276 Bialystok, Poland \\ Phone: +48 857468607 \\ Fax: +48 857447611 \\ E-mail: mszelachowska@ \\ poczta.onet.pl
}


The prevalence of LADA among type 2 diabetics in a large cohort of European individuals aged 25 to 65 years in the United Kingdom Prospective Diabetes study (UKPDS) was 10\% [2]. In Finish and Swedish individuals with type 2 diabetes, the prevalence was $9 \%$ and $8 \%$, respectively $[3,4]$. The incidence of LADA in Wales was calculated to be 9 cases per 100000 inhabitants, per year [5]. Identification of patients with LADA has important clinical implications, because these individuals require therapeutic intervention adjusted for the progressive decline of $\beta$ cell function [6-8].

We used the immunological marker anti-glutamic acid decarboxylase antibody (GADA) to identify LADA as this is the common standard used to identify patients with LADA. The GADA persists after diagnosis, and unlike ICA, commercial available assays can be used for measurements $[9,10]$. In this study we investigated patients with new onset diabetes and used an additional marker, anti-insulin antibody (IAA), to identify those with LADA.

Anti-insulin antibody were first identified in the sera of newly diagnosed insulin-naivve patients with type 1 diabetes by Palmer et al. [11]. Anti-insulin antibody has now been demonstrated as an autoimmune marker for prediction and diagnosis of type 1 diabetes [12, 13]. According to the Immunology of Diabetes Society, IAA can also be used to identify patients with LADA [1]. In a large Chinese population, IAA was found to be present (3.39\%) in phenotypic type 2 diabetes patients and, in combination with GADA, significantly increased the number of patients diagnosed with LADA [14].

The aim of this study was to determine the incidence and characteristics of LADA in a populationbased cohort of Polish patients with newly-diagnosed diabetes and determine whether anti-insulin antibody may be useful in identifying patients with LADA.

\section{Material and methods}

\section{Participants}

The study protocol was approved by the Ethics Committee of the Medical University of Bialystok. Informed consent was obtained from all patients before blood sampling. Diabetes was diagnosed according to the World Health Organization (WHO) 1999 criteria. The study base was the resident population of the city Bialystok, Poland, aged 20 to 64 years during the period 1 January 2003 to 31 December 2003 (291 931 inhabitants, 188738 aged 20-65 years). All cases of diabetes arising during the study period were identified through primary care physicians or primary care physicians and diabetologists working in the public health clinic. All patients were Polish citizens and, irrespective of their social status or employment, were cared for by a general practitioner as part of the National Health System. Primary care for diabetic patients is mainly provided by specialized diabetes clinics. All diabetic patients can obtain drugs and diabetes devices free of charge from the National Health System, provided they have certification of diabetes diagnosed. For the above-mentioned reasons, almost all newly diagnosed patients are attended by a primary care physician from the National Health System or diabetes clinic at the onset of the disease. Diabetologists and primary care physicians were periodically contacted by the coordinating centre. The coordinating center was the Department of Internal Medicine, Diabetology and Endocrinology, located in the Teaching Hospital of the Medical University of Bialystok. In 2003 and 2004, the clinical charts of all diabetes patients in the area of interest were reviewed to identify cases that had not been reported to the coordinating center and to assess clinical features of the individuals who had not been tested.

\section{Data collection}

Recruited participants arrived at the research unit for data collection after an overnight fast. Blood samples for biochemical and immunological analysis were obtained prior to the morning insulin or oral therapy. Detailed demographic data and clinical and treatment history were recorded using an interviewer-administered questionnaire. The data were verified using medical records. Anthropometric measurements were recorded according to the standard methods. Blood sampling was performed following diagnosis and stored at $-20^{\circ} \mathrm{C}$ for future analysis.

\section{Laboratory measurements}

Fasting plasma glucose was measured immediately by the enzymatic method using a glucose analyser. Total plasma cholesterol, HDL cholesterol and triglycerides were evaluated by the enzymatic method (Cormay, Poland); LDL cholesterol was calculated using Friedewald's formula. Glycated hemoglobin $\left(\mathrm{HbA}_{1 \mathrm{c}}\right)$ was assessed by the high performance liquid chromatography method (Bio-Rad, Germany). Plasma insulin was measured with the Medgenix EASIA test (Biosource Europe, Belgium). The minimum detectable concentration was 1.05 pg/l. In this method, human and animal proinsulin exhibits no cross-reactivity. C-peptide concentration was measured using an immunoenzymatic assay (Biosource Europe, Belgium) (specificity: $88 \%$, human proinsulin $-12.5 \%$, sensitivity: $0.01 \mathrm{pmol} / \mathrm{ml}$; intra-assay CV: 5.8\%). GADA and IAA were measured by commercially available RIA sets (CIS bio international, France). Anti-glutamic acid decarboxylase antibody was considered positive with 
a value higher than $1 \mathrm{U} / \mathrm{ml}$ according to recommendations for the kit. Binding percentage of ${ }^{125}$ insulin higher than mean value \pm 3 SD was considered positive for IAA. Available RIA commercial kits were used for GADA and IAA measurements. Beta cell function and insulin sensitivity were estimated by homeostatic model assessment (HOMA2).

\section{Terms and classifications}

Patients were classified as having type 1 diabetes if insulin treatment was started within 6 months of diagnosis. Diagnosis of LADA was made according to the criteria proposed by the Immunology Diabetes Society and Action LADA [1]. Those who were aged $\geq 30$ years at diagnosis, autoantibody positive, and insulin independent during the first 6 months after diagnosis were considered to have LADA. Participants who were GADA or IAA negative and insulin independent for 6 months or more after diagnosis were considered to have type 2 diabetes.

\section{Statistical analysis}

The incidence was calculated as the number of new cases of disease in a defined group of people over a specific time. Data are shown as mean \pm SD. The Mann-Whitney $U$ test and Spearman correlation test were applied. A $p$ value of $<0.05$ was considered statistically significant.

\section{Results}

\section{Incidence rate of diabetes in Bialystok population}

In 2003, 231 cases of newly diagnosed diabetes in a population of 188738 inhabitants in the 20 to 65 years age range were found. The incidence of known diabetes in this population was therefore 1.22 per 1000 per year (95\% confidence interval (Cl) 1.07-1.39 per 1000 per year).

\section{Characteristics of the study participants}

Two hundred and twelve patients (212) participated in the study. This represents $91 \%$ of the sample collection. Among 212 participants, 54.2\% were males. The mean $\mathrm{HbA}_{1 \mathrm{c}}$ was $7.2 \pm 1.9 \%$. The mean body mass index (BMI) in the whole diabetic population was $29.2 \pm 5.4 \%$.

\section{Percentage of GADA/IAA autoantibody positivity}

The percentage of GADA/IAA-positive patients in this cohort was $10.3 \%(n=22 ; 95 \% \mathrm{Cl} 0.66-1.54)$. Anti-glutamic acid decarboxylase antibody was present in $7.0 \%(n=15 ; 95 \% \mathrm{Cl} 4.11-11.4)$, and IAA in $4.5 \%(n=9 ; 95 \% \mathrm{Cl} 2.07-7.79)$ of people with newly diagnosed diabetes.

\section{Clinical features associated with GADA/IAA autoantibody positivity}

In all groups, the presence of autoantibody was correlated with the age at diagnosis $(r=-0.187$, $p<0.05)$, body mass index (BMI) $(r=-0.182$, $p<0.05)$, waist $(r=0.214, p<0.05)$, fasting $C$ peptide $(r=-0.300, p<0.0001)$, fasting insulin $(r=-0.213, p<0.0001)$, HOMA 2\% B $(r=-0.219$, $p<0.01)$, HOMA2-IR $(r=0.303, p<0.001)$.

\section{Subtypes of diabetes}

In our cohort, $87.7 \%(n=186 ; 95 \% \mathrm{Cl} 7.57-10.1)$ were diagnosed with type 2 diabetes. Latent autoimmune diabetes in adults was diagnosed in $8.9 \%$ ( $n=19 ; 95 \% \mathrm{Cl} 5.55-13.74)$. Subtype of diabetes in relation to auto-antibodies: the majority (63\%) of patients with LADA had anti-GAD antibodies ( $n=12)$; IAA were present in eight patients with LADA. One patient had both antibodies GADA and IAA. Up to $36 \%$ of patients diagnosed with LADA had IAA present.

The diagnosis of type 1 diabetes was made in $3.3 \%$ of individuals $(n=7 ; 95 \% \mathrm{Cl} 1.44-6.54)$. Among them, three subjects were positive for autoimmune antibody ( $n=2$ GADA, $n=1$ both GADA and IAA) and four negative.

\section{Characteristics of LADA and type 2 diabetes}

Patients with LADA were compared to those with type 2 diabetes and were found to be younger at the time of diagnosis, had lower BMI, waist circumstance, fasting $C$ peptide level and insulin, lower beta cell function (HOMA 2\%B) and at the same time were less insulin resistance than patients with type 2 diabetes (HOMA2-IR). Characteristics of LADA and type 2 diabetes are depicted in Table I.

\section{Discussion}

This is the first study to examine the epidemiology of LADA in the Polish population. We identified and tested more than $90 \%$ of newly diagnosed cases of diabetes during 1 year in the Bialystok area. The incidence of LADA was 10 per 100000 per year and was similar to that described for the population living in South Wales (9 per 100000 per year) [5].

The proportion of LADA among newly diagnosed patients with diabetes was $8.9 \%$. Taking into account that in this study the majority of patients were diagnosed with type 2 diabetes, the observed proportion is similar to that found in the UKPDS, Finish and Swedish studies [2-4], and higher than detected in the Horn study, mainly due to the different age of the population tested [15].

Compared to patients with type 2 diabetes, patients with LADA were younger at the time of diagnosis, had lower BMI, waist circumstance, 
Table I. Clinical and metabolic features of patients with LADA and type 2 diabetes in a population-based cohort of newly diagnosed people with diabetes

\begin{tabular}{|lccc|}
\hline Parameter & LADA & $\begin{array}{c}\text { Type } 2 \\
\text { diabetes }\end{array}$ & Value of $p$ \\
\hline Number & 19 & 186 \\
\hline $\begin{array}{l}\text { Age at diagnosis } \\
\text { [years] }\end{array}$ & $48.5 \pm 9.4$ & $54.8 \pm 10.6$ & $<0.01$ \\
\hline BMI [kg/m²] & $26.8 \pm 9.3$ & $29.5 \pm 5.2$ & $<0.05$ \\
\hline Gender M/F & $9 / 13$ & $103 / 87$ & NS \\
\hline $\begin{array}{l}\text { Waist circumstance } \\
\text { [cm] }\end{array}$ & $90.5 \pm 12.7$ & $100.6 \pm 13.5$ & $<0.05$ \\
\hline $\begin{array}{l}\text { Fasting glucose } \\
\text { [mmol/l] }\end{array}$ & $189.9 \pm 108.3$ & $160.1 \pm 69.9$ & NS \\
\hline HbA 1 lc $\%$ ] & $7.86 \pm 3.1$ & $7.22 \pm 1.7$ & NS \\
\hline $\begin{array}{l}\text { Fasting C peptide } \\
\text { [pmol/l] }\end{array}$ & $126.4 \pm 127.9$ & $446.3 \pm 592.2$ & $<0.001$ \\
\hline $\begin{array}{l}\text { Fasting insulin } \\
\text { [pmol/l] }\end{array}$ & $81.2 \pm 64.5$ & $98.6 \pm 58.3$ & $<0.05$ \\
\hline $\begin{array}{l}\text { Total cholesterol } \\
\text { [mg/dl] }\end{array}$ & $205.5 \pm 50.7$ & $210.5 \pm 46.7$ & NS \\
\hline HDL-C [mg/dl] & $50.1 \pm 15.6$ & $45.6 \pm 13.0$ & NS \\
\hline LDL-C [mg/dl] & $139.3 \pm 37.9$ & $132.5 \pm 34.8$ & NS \\
\hline TG [mg/dl] & $126 \pm 46.9$ & $153.6 \pm 98.4$ & NS \\
\hline HOMA 2\%B & $45.7 \pm 52.7$ & $109 \pm 127$ & $<0.05$ \\
\hline HOMA IR & $0.94 \pm 0.85$ & $3.6 \pm 4.4$ & $<0.001$ \\
\hline
\end{tabular}

Data are presented as mean and SD. The $p$ values were calculated using the Mann-Whitney $U$ test

C peptide levels and were less insulin resistant. These observations are concordant with other studies regarding LADA [16-18]. Patients with newly diagnosed LADA presented with similar fasting glucose and $\mathrm{HbA}_{1 \mathrm{c}}$ compared to patients with type 2 diabetes, suggesting rather slow progression and relatively asymptomatic clinical onset [17].

To identify individuals with LADA we used both GADA and anti-insulin antibody. We tested IAA in the newly diagnosed population, before insulin treatment was introduced. In our study, $4.5 \%$ of all patients with newly diagnosed diabetes and 36\% of patients with LADA were positive for IAA. This percentage is lower than in patients with newly diagnosed type 1 diabetes $[13,19]$. In the study by Huang et al. [14] Anti-insulin autoantibodies was present in $3.39 \%$ of 100 patients with type 2 diabetes. Anti-insulin autoantibodies when tested at the time of diagnosis may be useful in identifying patients with LADA.

This study, however, was limited to the urban population in the city of Bialystok, and it should be noted that in Poland there are differences in diabetes epidemiology between urban and rural populations. In the EURODIAB TIGER programme the incidence rate for type 1 diabetes was higher in the rural population, mostly due to rapidly increasing socio-economic status and change in lifestyle. In the future, it will be interesting to compare the incidence of LADA between rural and urban populations [20].

In conclusion, LADA presents with similar clinical onset to type 2 diabetes with significant beta cell dysfunction and lower insulin resistance. In addition to GADA, IAA can also be a useful marker in identifying adults with autoimmune diabetes, before insulin treatment is introduced. In the Polish population, adult autoimmune diabetes accounts for a significant proportion of patients with diabetes mellitus. In this context, screening patients with type 2 diabetes, especially younger and with lower BMI level, may be a worthy effort to screen for LADA and offer these individuals treatment in concordance with declining beta cell function [16, 21].

\section{Acknowledgments}

We thank the patients, diabetes clinic nurses, diabetologists, and general practitioners in Bialystok for providing important collaboration to this study. We thank Garbriela Kozłowska, Elżbieta Otziomek and Agnieszka Nikołajuk for their invaluable assistance with this study.

We declare that there is no conflict of interest that could be perceived as prejudicing the impartiality of the research reported. This work was supported by a research grant for the Medical University of Bialystok (grant number 3-50956).

\section{References}

1. Leslie RD, Kolb H, Schloot NC, et al. Diabetes classification: grey zones, sound and smoke: Action LADA 1. Diabetes Metab Res Rev 2008; 24: 511-9,

2. Turner R, Stratton I, Horton V, et al. UKPDS 25: autoantibodies to islet-cell cytoplasm and glutamic acid decarboxylase for prediction of insulin requirement in type 2 diabetes. UK Prospective Diabetes Study Group. Lancet 1997; 350: 1288-93.

3. Wroblewski M, Gottsater A, Lindgarde F, Fernlund P, Sundkvist G. Gender, autoantibodies, and obesity in newly diagnosed diabetic patients aged $40-75$ years. Diabetes Care 1998; 21: 250-5.

4. Tuomi T, Carlsson A, Li H, et al. Clinical and genetic characteristics of type 2 diabetes with and without GAD antibodies. Diabetes 1999; 48: 150-7.

5. Davies H, Brophy S, Fielding A, et al. Latent autoimmune diabetes in adults (LADA) in South Wales: incidence and characterization. Diabet Med 2008; 25: 1354-7.

6. Gottsater A, Landin-Olsson M, Fernlund P, Lernmark A, Sundkvist $\mathrm{G}$. Beta-cell function in relation to islet cell antibodies during the first $3 \mathrm{yr}$ after clinical diagnosis of diabetes in type II diabetic patients. Diabetes Care 1993; 16: 902-10.

7. Torn C, Landin-Olsson M, Lernmark A, et al. Prognostic factors for the course of beta cell function in autoimmune diabetes. J Clin Endocrinol Metab 2000; 85: 4619-23. 
8. Brophy S, Brunt H, Davies H, Mannan S, Williams R. Interventions for latent autoimmune diabetes (LADA) in adults. Cochrane Database Syst Rev 2007; CD006165.

9. Davies H, Mannan S, Brophy S, Williams R. Routine glutamic acid decarboxylase autoantibody (GADA) testing: patients' perspective. Fam Pract 2008; 25: 176-80.

10. Desai M, Cull CA, Horton VA, et al. GAD autoantibodies and epitope reactivities persist after diagnosis in latent autoimmune diabetes in adults but do not predict disease progression: UKPDS 77. Diabetologia 2007; 50: 2052-60.

11. Palmer JP, Asplin CM, Clemons P, et al. Insulin antibodies in insulin-dependent diabetics before insulin treatment. Science 1983; 222: 1337-9.

12. Hummel M, Bonifacio E, Schmid S, Walter M, Knopff A, Ziegler AG. Brief communication: early appearance of islet autoantibodies predicts childhood type 1 diabetes in offspring of diabetic parents. Ann Intern Med 2004; 140: 882-6.

13. Eisenbarth GS, Moriyama H, Robles DT, et al. Insulin autoimmunity: prediction/precipitation/prevention type 1 A diabetes. Autoimmun Rev 2002; 1: 139-45.

14. Huang G, Wang X, Li Z, Li H, Li X, Zhou Z. Insulin autoantibody could help to screen latent autoimmune diabetes in adults in phenotypic type 2 diabetes mellitus in Chinese. Acta Diabetol 2010 May 16. [Epub ahead of print].

15. Ruige JB, Batstra MR, Aanstoot HJ, et al. Low prevalence of antibodies to GAD65 in a 50- to 74-year-old general Dutch population. The Hoorn Study. Diabetes Care 1997; 20: 1108-10.

16. Carlsson A, Sundkvist G, Groop L, Tuomi T. Insulin and glucagon secretion in patients with slowly progressing autoimmune diabetes (LADA). J Clin Endocrinol Metab 2000; 85: 76-80.

17. Stenstrom G, Gottsater A, Bakhtadze E, Berger B, Sundkvist $\mathrm{G}$. Latent autoimmune diabetes in adults: definition, prevalence, beta-cell function, and treatment. Diabetes 2005; 54 Suppl 2: S68-72.

18. van Deutekom AW, Heine RJ, Simsek S. The islet autoantibody titres: their clinical relevance in latent autoimmune diabetes in adults (LADA) and the classification of diabetes mellitus. Diabet Med 2008; 25: 117-25.

19. Williams AJ, Norcross AJ, Dix RJ, Gillespie KM, Gale EA, Bingley PJ. The prevalence of insulin autoantibodies at the onset of Type 1 diabetes is higher in males than females during adolescence. Diabetologia 2003; 46: 1354-6.

20. Szurkowska M, Szybinski Z, Nazim A, Szafraniec K, Jedrychowski W. Prevalence of type II diabetes mellitus in population of Krakow [Polish]. Pol Arch Med Wewn 2001; 106: 771-9.

21. Berger B, Borg H, Fernlund P, Stenstrom G, Sundkvist G. Islet antibodies associated with pancreatic B-cell dysfunction at and 3 years after diagnosis of diabetes in subjects aged 35-64 years old: degree of impairment less severe than in those aged 0-34 years old. Diabet Med 2006; 23: 1180-5. 